\title{
OPTIMIZED FLOODING AND INTERFERENCE-AWARE QOS ROUTING IN OLSR*
}

\author{
Dang Quan Nguyen ${ }^{1}$ and Pascale Minet ${ }^{1}$ \\ ${ }^{1}$ INRIA Rocquencourt, Domaine de Voluceau \\ Rocquencourt - B.P. 105 \\ 78153 Le Chesnay Cedex, France \\ dang-quan.nguyen@inria.fr, pascale.minet@inria.fr
}

\begin{abstract}
Radio interferences and low capacity resources in ad-hoc wireless networks make more complex the quality of service (QoS) support. We propose a solution taking into account radio interferences in mobile ad-hoc networks routing and providing an optimized flooding based on multipoint relays. This solution is based on a modified version of the OLSR routing protocol that considers bandwidth requests and interferences in route selection while providing a very efficient flooding. A comparative performance evaluation based on NS simulations shows that despite the overhead due to QoS support, this solution outperforms classical OLSR in terms of maximum number of acceptable flows, bandwidth amount granted to a flow and route stability. Moreover, the efficiency of the optimized flooding is equal to that provided by the native version of OLSR.
\end{abstract}

Keywords: Interference, quality of service, optimized flooding, QoS routing, OLSR, multipoint relay (MPR).

\section{Introduction}

In a MANET, QoS support is harder than in a wired network because of interferences. The transmission of a node is said to interfere with the transmission of other nodes if at the receivers, the carrier to interference ratio is lower than a threshold value.

Another diffi culty of QoS support in MANETs is that they are characterized by low capacity time-varying resources. The bandwidth of already accepted fbws may signifi cantly decrease as a new fbw is introduced. That is why an admission control is needed. Both the admission control and QoS routing must

\footnotetext{
*This study has been funded by DGA/CELAR (French Ministry of Defence), in MANET project. Technical point of contact: thierry.plesse@dga.defense.gouv.fr
} 
be interference aware. Furthermore, disseminating information (e.g.; network topology in a proactive routing protocol) or information request (e.g.; route request in a reactive routing protocol) is frequently needed. In order to avoid resources wastage, optimized fboding is required. A solution based on multipoint relays (MPR) is more adaptive than a solution based on a predefi ned connected dominating set and leads to less retransmissions. As a consequence, a solution based on OLSR routing providing QoS support and optimized fboding is needed. This is the purpose of this paper.

\section{Interference aware QoS OLSR}

In [3], it is shown that fi nding a path from a source to a destination that satisfi es a bandwidth constraint is made NP-complete by the interferences. That is why we propose a heuristic.

\subsection{General presentation}

We choose a QoS routing algorithm based on OLSR where the hop count is the primary criterion and the local available bandwidth is the secondary one, for the following reasons:

- The shortest routes, having the minimum hop count, tend to minimize the network resources used for the transmission of a packet from its source to its destination. That is why the hop count must be taken into account in order to reduce the bandwidth loss due to interferences.

- Some fbws have bandwidth requirements. Hence the local available bandwidth must be taken into account in the route selection.

- Being called upon any topology change, the chosen routing algorithm must have a complexity similar to Dijkstra algorithm.

- Resources in a MANET having low capacity, the chosen algorithm is based on a partial topology knowledge, like OLSR.

QoS parameters values are disseminated in the network by means of MPRs. The selection of MPRs is modifi ed to consider the bandwidth locally available at each node. The main drawback of this solution lies in the overhead generated: each fboded message leads to a number of retransmissions higher than that obtained with the native OLSR. In this paper, we show how to conciliate the optimized performances of MPR fboding with QoS support. For that purpose, we distinguish two types of MPRs:

- Those, called MPRF, are selected according to the native version of OLSR and are used to optimize fboding.

- The other ones, called MPRB, are selected considering the local available bandwidth and are used to build the routes.

We now detail each component of our solution. 


\subsection{QoS signaling}

Measure of the local available bandwidth. The Local Available Bandwidth (LAB) of a node is measured locally at the MAC level: the percentage of time the channel is sensed idle and the node is not in back-off state, multiplied by the channel capacity.

Dissemination of the bandwidth available. Any node broadcasts its LAB as well as the LAB of its one-hop neighbors in its Hello message. Consequently any node knows the LAB of each node in its one-hop neighborhood and two-hop neighborhood.

Any MPRB node broadcasts in its TC message the LAB of its MPRB selectors and the minimum LAB in its interference area. Notice that according to OLSR rule, only MPRF nodes of the sender retransmit the received message and only if they receive it for the fi rst time.

\subsection{MPR selection}

Each node performs two MPR selections. The fi rst one is to determine the MPRFs. It is done as specifi ed in the OLSR RFC. The second one is to determine the MPRBs. Knowing the LAB of its neighbors and two-hop neighbors, any node $N_{i}$ selects its MPRBs in order to reach each two-hop neighbor by a path of maximum bandwidth. If for a two-hop neighbor, there are several one-hop neighbors reaching it, the one with the highest LAB is selected.

The Hello message sent by a node contains both its MPRFs and its MPRBs. A node selected as MPRB broadcasts a TC.

\subsection{QoS routing}

Two QoS routing algorithms are used. Algorithm 1, unconstrained, aims at providing the widest shortest path between any two nodes (i.e.; the path with the minimum hop number and in case of equality, the path with the largest available bandwidth). It is the default algorithm used to compute the routing table. On the other hand, Algorithm 2, constrained by a bandwidth request, is used to compute a route offering the requested bandwidth to reach a destination. It is called by the admission control for a new flow $f$ requesting $B_{f}$ bandwidth units.

\subsection{Admission control}

The purpose of an admission control is to accept a new fbw $f$ if and only if the QoS of already accepted fbws is not compromized by the acceptance of $f$ and the QoS requested by $f$ can be met. It checks that all nodes on the path of 
$f$ provide the requested bandwidth and any node in the interference area of a node on the path of $f$ has enough bandwidth.

\section{Performance evaluation}

We evaluate the performance of our solution by simulation with NS. According to the default value of NS-2, the reception range of a node is $250 \mathrm{~m}$ and the carrier-sense range is $550 \mathrm{~m}$. We use the IEEE $802.11 \mathrm{~b}$ MAC protocol without RTS/CTS. The nominal bandwidth is $11 \mathrm{Mbps}$. OLSR parameters are set as recommended in [1].

\subsection{Flooding optimization}

We consider a network with 200 nodes, randomly located on a $2000 \times 2000 \mathrm{~m}^{2}$ area. Figure 2 shows that our solution with MPRB-optimized generates as many TCs as MPRB that is used by QOLSR [4]. Notice that this is necessary for computing paths with maximum bandwidth. The interesting point is that our solution maintains the number of retransmissions per TC generated as low as MPRF. Therefore, MPRB-optimized performs effi cient fboding.

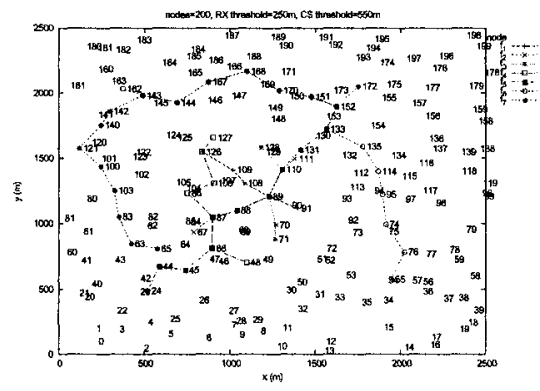

Figure 1. Ad hoc network with 200 nodes.

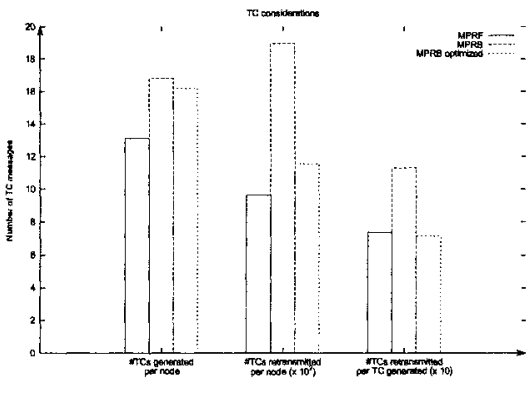

Figure 2. TC considerations, 200 nodes.

\subsection{QoS support}

Configuration studied. We now simulate 7 CBR fbws with this configuration (see Figure 1). The fbws are CBR. Each fbw requires $175 K$ bps at the application layer. The packet size is 1500 bytes.

Bandwidth granted to flows. Figures 3 and 4 respectively show the bandwidth received at the application layer of each fbw in the two following scenarios: $7 \mathrm{fbws}$ with Interference-Aware routing and $7 \mathrm{fbws}$ with the native OLSR protocol. The simulations show that Interference-Aware routing can provide bandwidth guarantee to QoS fbws when the bandwidth resource is still available. The native OLSR cannot offer this guarantee because it does not take into account the availability of bandwidth resource. 
With our solution, we can notice that in Figure 1, fbws 6 and 7 are routed around the center of the network because there is not enough bandwidth available in the center to support them. Routing fbws 6 and 7 across the center of the network would degrade the bandwidth already granted to the other fbws as shown in Figure 4.

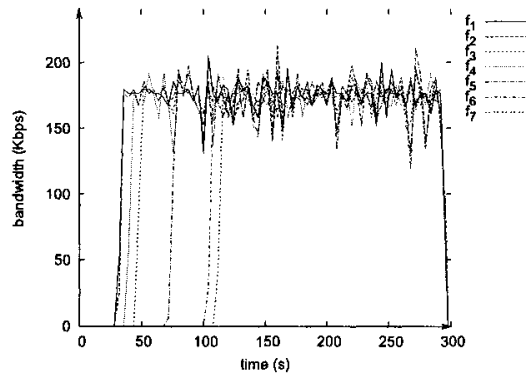

Figure 3. Interference-Aware routing.

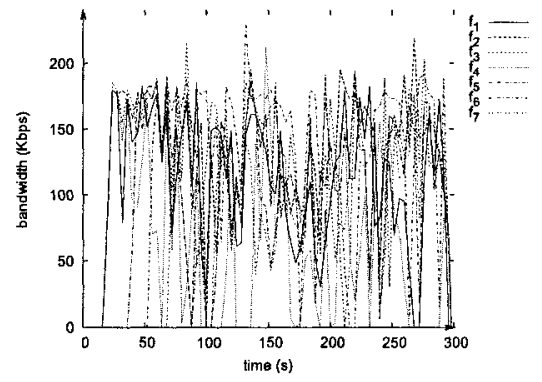

Figure 4. Native OLSR protocol.

Moreover, our solution achieves much less packet loss. Indeed, the loss rates averaged on all fbws and measured at the application level of each $\mathrm{fbw}$ destination is equal to $53.08 \%$ with native OLSR whereas it is only $1.85 \%$ with our solution.

\section{Conclusion}

In this paper, we have shown how to conciliate an interference-aware QoS support with an effi cient fboding. Our solution distinguishes two types of multipoint relays: those in charge of MPR fboding that are selected as specifi ed in the classical OLSR version and the other ones that are used to select routes considering bandwidth demand and interferences. Simulation results have shown that the proposed solution allows to accept more fbws, routes are more stable and accepted fbws receive the bandwidth they have requested. Moreover, the overhead due to this QoS support, is kept low and fboding achieves the very good performances of native OLSR.

\section{References}

[1] C. Adjih, T. Clausen, P. Jacquet, A. Laouiti, P. Minet, P. Muhlethaler, A. Qayyum, L. Viennot: Optimized Link State Routing Protocol, RFC 3626, IETF, 2003.

[2] Y. Ge, T. Kunz, L. Lamont: Quality of Service Routing in Ad-Hoc Networks Using OLSR, HICSS'03, Big Island, Hawai, January 2003.

[3] G. Allard, L. Georgiadis, P.Jacquet, B. Mans: Bandwidth Reservation in Multihop Wireless Networks: complexity, heuristics and mechanisms, International Journal of Wireless and Mobile Computing (inderscience), accepted for publication in May 2004, To appear (ISSN-1741-1084).

[4] H. Badis and K. Al Agha: QOLSR, QoS routing for Ad Hoc Wireless Networks Using $O L S R$, in European Transactions on Telecommunications, vol. 15, $\mathrm{n}^{\circ} 4,2005$. 